\title{
Ciclos de interesse coletivo e tendências das buscas no Google relacionadas a campanhas institucionais sobre o câncer de próstata: promovendo saúde ou doenças?
}

\author{
Collective interest cycles and trends in Google searches \\ related to institutional campaigns on prostate cancer: \\ promoting health or illness?
}

${ }^{1}$ Instituto Nacional de Câncer, Coordenação de Pesquisa. R. André Cavalcanti 37, Centro 20231-050. Rio de Janeiro RJ Brasil. p.vasconcellos@pq.cnpq.br ${ }^{2}$ Instituto Oswaldo Cruz, Fundação Oswaldo Cruz. Rio de Janeiro RJ Brasil.

\begin{abstract}
Google algorithms record trends in interest on topics relevant to public health. WEB searches were analyzed (2014-2019) to identify patterns linked to prostate cancer. Relative Search Volumes (RSV) were analyzed by Google Trends on "prostate cancer" (PC), "prostate examination" (PE) and "PSA"; 260-week time series; Brazil region; Health category; Trend lines (degree 2 polynomials) to identify patterns; Averages compared by ANOVA; Sudden increase in November searches; Searches on PC greatly surpass PE and PSA; Stable annual PC averages; Discreet reduction in PE; Marked increase in PSA. In campaign months: Discreet increase in PC; stability in PE; Marked increase in PSA. "Blue November" campaigns encourage early identification of prostate cancer, although interest is seen to be focused on the disease per se with a lack of interest in diagnosis throughout the year. Differences in relation to "Pink October" are discussed - tenuous relation to educational level on prevention habits and the influence of celebrities. The conclusion drawn is that RSV analysis might be useful in tracking trends in prostate cancer screening to provide input for campaign developers.

Key words Early detection of cancer, Information-seeking behavior, Prostate cancer, Health promotion, Internet and health
\end{abstract}

Resumo Algoritmos do Google registram tendências no interesse sobre temas relevantes à saúde coletiva. Analisamos buscas na web (2014-2019) para identificar padrões ligados ao câncer de próstata. Analisamos volumes de pesquisa relativos (VPR) pelo Google Trends sobre "câncer de próstata" (CAP), "exame de próstata"(EP) e "PSA"; séries temporais de 260 semanas; região Brasil; categoria "saúde" e linhas de tendência (polinomiais ordem 2) para identificar padrões; médias comparadas por ANOVA. Identificamos crescimentos súbitos nas buscas nos meses de novembro; buscas sobre CAP superam largamente EP e PSA; médias anuais CAP estáveis; EP com discreta queda; PSA com marcada ascensão. Nos meses de campanhas: elevação discreta de CAP; estabilidade em EP; marcada elevação de PSA. O "Novembro Azul" incentiva a identificação precoce do câncer de próstata, embora observe-se interesse focado na doença e desinteresse sobre o diagnóstico ao longo do ano. Diferenças em relação ao "Outubro Rosa" são discutidas - fraca interferência do nível de escolaridade sobre os hábitos de prevenção e influência de celebridades. Concluiu-se que análises de VPR podem ser úteis ao acompanhamento de tendências sobre o rastreio do câncer de próstata para fornecer subsídios aos desenvolvedores das campanhas.

Palavras-chave Detecção precoce de câncer, Comportamento de busca de informação, Câncer de próstata, Promoção de saúde, Internet e saúde 


\section{Introdução}

O câncer de próstata é o segundo tumor maligno mais frequente no Brasil e as estimativas indicam cerca de 68.220 novos casos em 2018, com 15.391 mortes em $2017^{1}$. A literatura especializada sobre a necessidade de rotina de rastreamento do câncer de próstata apresenta alguns dissensos ${ }^{2,3}$, embora haja consenso acerca da relação entre a detecção precoce e melhores probabilidades de cura. No entanto, muitos obstáculos se apresentam ao pleno sucesso das campanhas que buscam incentivar o diagnóstico precoce, como a objeção cultural ao exame clínico da próstata relacionada a tabus masculinos, assim como a aspectos de ordem estrutural dos sistemas de saúde ligados à dificuldade de acesso e diagnóstico ${ }^{4}$. É possível concluir, assim, que para tornar as campanhas institucionais plenamente exitosas em termos de vidas salvas e economia de recursos, deve-se considerar tanto os fatores que interferem no acesso, atenção integral e resolutividade dos serviços como conhecer e buscar intervir nos fatores que influenciam o interesse e motivação dos homens a buscar informações e agir na direção da identificação precoce do problema ${ }^{5,6}$.

Em relação a este último campo de pesquisa, os estudos se proliferam em número, diversificação de temáticas e estratégias para caracterizar o impacto das campanhas em termos de repercussões nas novas mídias. Percebe-se um interesse crescente de pesquisadores e formuladores de políticas públicas em analisar tendências de buscas na Internet para estimar sucessos e identificar obstáculos às mensagens institucionais ligadas à prevenção ou ao diagnóstico precoce de doenças. Em relação ao comportamento de prevenção adotado por casais idosos, por exemplo, sabe-se da relevância central da Internet como fonte de referência e suporte, sobretudo em contextos de mútuo interesse pela saúde ${ }^{7,8}$. $\mathrm{O}$ envolvimento comunitário também pode conferir impulso vigoroso às campanhas voltadas à saúde do homem, como descrito por estudos que analisaram postagens sobre esses assuntos pelo Twiter ${ }^{9,10}$.

$\mathrm{Na}$ era dos dados digitais amplamente acessíveis a grande parcela da população, usuários dos sistemas de saúde estão cada vez mais envolvidos em buscas reativas (movidas por curiosidade ou interesse descompromissado) ou proativas (focadas na efetiva mudança ou na expansão dos hábitos de prevenção) ${ }^{11}$, ou, como preferem outros autores, na information seeking behavior ${ }^{7,12}$. Em outros termos, o interesse que movimenta essas pesquisas pode se desdobrar em opções de pre- venção, diagnóstico ou tratamento, assim como retratar apenas curiosidade sobre a natureza e evolução de doenças como o câncer. Apesar de tantas informações disponíveis, tais procuras nem sempre se desdobram em prevention behavior (comportamento de prevenção) $)^{6,12,13} \mathrm{em}$ consequência das buscas proativas. Isso acrescenta o desafio de reconhecer padrões incrementais de buscas proativas para analisar os fatores que influenciaram os sucessos obtidos e assim planejar campanhas que se apropriem deles, incitando aos hábitos de prevenção ${ }^{13-16}$. Além disso, na dimensão do acesso aos serviços, nas regiões e nos períodos em que se perceba interesse crescente - por meio da identificação de buscas proativas que apontem tais tendências -, deve-se organizar apropriadamente as estruturas assistenciais para receber usuários que afluem em consequência de campanhas exitosas.

Sob ponto de vista das tecnologias de informação, há diversas formas de estimar em larga escala o sucesso (ou insucesso) de campanhas de rastreamento para estabelecer ilações pautadas em variáveis culturais implicadas nos resultados. A experiência com sistemas de informação dedicados à utilização dos serviços de rastreamento de câncer mostra que, embora de incalculável valor na formulação de hipóteses sobre variáveis condicionantes que impelem ao screening, há limitações insuperáveis ligadas aos elevados custos investidos no desenvolvimento e implantação desses sistemas ${ }^{16}$. Por outro lado, um número massivo de consultas no Google de usuários interessados nesses temas gera dados que podem ser analisados instantaneamente pelo Google Trends (GT) - recurso de acesso público on-line usado para acompanhar tendências do volume de pesquisas na web ${ }^{17}$.

O Google Trends tem sido usado desde 2006 como fonte de identificação de ciclos de interesse que movimentam o consumo de bens, ideias e modismos vinculados a ordenamentos culturais de um meio social. Indo muito além do consumo de fruição, percebeu-se que retrata também o consumo de informações relacionados a surtos epidêmicos em fase inicial ${ }^{18,19}$; o interesse coletivo pelo rastreio de doenças ${ }^{13,20}$; e o modismo vinculados à preservação da beleza e da juventude ${ }^{21,22,}$ entre muitas outras questões ligadas à saúde coletiva. Aos planejadores de políticas públicas, o GT pode fornecer dados de feedback essenciais acerca do volume de consultas vinculadas às questões que se deseja estudar quase imediatamente após as intervenções. Acredita-se que as variações no volume de buscas mais expressivas podem se 
relacionar a ciclos de interesse coletivo influenciados por campanhas entre outros elementos culturalmente substantivos ${ }^{11,23}$. Como sugerem alguns autores, podem ser úteis na "previsão do presente" 24 ao caracterizar oscilações das queries pelo Google associáveis ao consumo de informações sobre matérias de interesse coletivo ${ }^{25}$.

No presente trabalho, estudamos oscilações no volume de consultas no Google voltadas a termos ligados a doenças como "câncer de próstata", comparados a termos ligados ao seu rastreamento - "exame de próstata" e "PSA" -, buscando identificar e analisar padrões de buscas e suas evoluções após as campanhas do Novembro Azul ao longo dos últimos cinco anos.

\section{Metodologia}

O Google Trends (https://www.google.com.br/ trends) é um recurso de acesso público on-line que mensura o volume de pesquisas acerca de determinados assuntos, segmentando-o por países/ regiões, temas gerais, período das buscas e formas de veiculação das informações (imagens, notícias, vídeos e propaganda comercial). Foram estudados os volumes de buscas realizadas no Brasil de agosto de 2014 a agosto de 2019, concentradas no tema "saúde" e em "toda a eeb". Os algoritmos do Google Trends normalizam os dados do número total de pesquisas em uma escala de 0 (volume de pesquisa inferior a $1 \%$ do volume de pico) a 100 (pico de popularidade), apresentando-as como um volume de pesquisa relativo (VPR) semanal. Os valores de VPR são representados de 0 a 100, retratando uma estimativa de proporção comparada ao VPR mais alto. Esses recursos metodológicos corrigem os resultados adequando o tamanho da população e o volume de acesso às regiões de onde são extraídos.

Os termos de busca foram escolhidos por sua associação semântica com a descrição de doenças e seus sintomas, em comparação relativa com termos derivados, usualmente encontrados nas primeiras procuras e aqui atribuídos à busca por informações sobre "o que fazer" após conhecer as doenças e alternativas de diagnóstico dessas condições. Tal critério segue lógica semelhante à de Schootman ${ }^{16}$ e Vasconcellos-Silva ${ }^{13}$, que empregaram palavras que diferenciassem interesse sobre a doença, separando-as dos termos ligados à efetiva preparação para os exames (como "câncer de cólon" e "câncer de mama", representantes da primeira categoria, e "miralax" - substância usada na preparação para colonoscopias - e "mamo- grafia", representantes da última). O termo usado como proxy das buscas ligadas ao primeiro grupo foi "câncer de próstata". Para buscas voltadas ao interesse no diagnóstico precoce da doença, usamos "exame de próstata" "PSA" (prostate specific antigen). Os termos "exame de PSA" e "dosagem de PSA", entre outros, foram preteridos, em vista do pequeno volume de procuras em comparação a "PSA". Este, além de apresentar VPR maiores, mostrou em seus ciclos certa "sincronia" com os demais ciclos ligados à temática geral. A seleção do termo "exame de próstata" seguiu a mesma lógica para dispensar termos assemelhados.

Foram produzidas séries temporais (superpostas na Gráfico 1) de todos os termos de busca, representando 260 semanas - de agosto de 2014 a agosto de 2019 -, com filtros ligados ao país - "Brasil" (país) - e pertencentes à categoria "saúde". Os resultados fornecidos pelo GT como arquivos CSV foram exportados para planilhas e as diferenças entre as curvas foram analisadas por ANOVA (análise de variância), com médias de VPR semanais e mensais comparadas às médias anuais correspondentes. Um gráfico da série temporal de cinco anos foi traçado com registro de todas as VPR semanais, além das linhas de tendência polinomiais (ordem 2), adicionadas para estimar tendências ao longo do tempo.

Considerações éticas: os dados disponibilizados pelo Google Trends são de domínio público, o que dispensaria o projeto de apreciação ética de acordo com a resolução 510/2016 do Conselho Nacional de Saúde ${ }^{26}$ e com a Declaração de Helsinque $^{27}$.

\section{Resultados}

Em termos gerais, ao longo das 260 semanas registradas, as buscas direcionadas a "câncer de próstata" superam por larga vantagem as voltadas a "exame de próstata" e "PSA". Na Gráfico 1 são registrados os acessos aos três termos, assim como a linha de tendência relativa a "câncer de próstata". Torna-se evidente o efeito de "achatamento" relativo de "câncer de próstata" sobre os demais termos de busca. De forma geral, o mesmo efeito é observado sempre que um volume massivo de buscas é comparado a outros bem menos "populares".

Em relação a todos os termos, observa-se os cinco picos de interesse nos meses de novembro, em meio à VPR homogeneamente distribuídos (Tabela 1 e gráficos 1, 2 e 3). Como evidente na Gráfico 3, o efeito do Novembro Azul é menos 


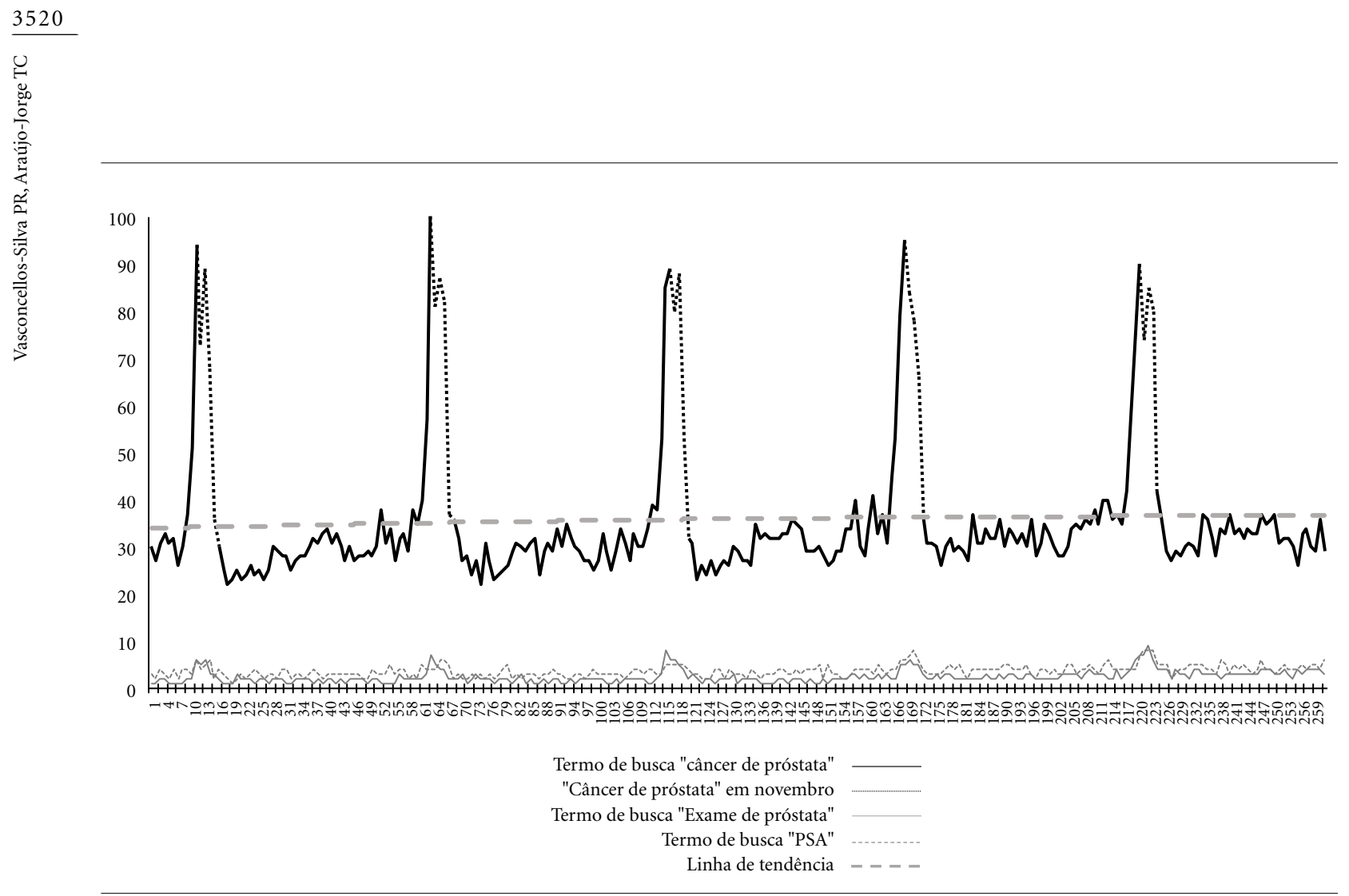

Gráfico 1. Oscilações dos termos de busca ao longo de 261 semanas.

Fonte: Google Trends (https://trends.google.com/trends/explore?cat=45\&date=today\%205-y\&geo=BR\&q=cancer\%20de\%20pr\%C3\%B3stata; https:// trends.google.com/trends/explore?cat=45\&date=today $\% 205-\mathrm{y} \& g e o=B R \& q=$ psa; ehttps://trends.google.com/trends/explore?cat=45\&date $=$ today $\% 20$ 5 -y\&geo= BR\&q=exame $\% 20 \mathrm{de} \% 20 \mathrm{pr} \% \mathrm{C} 3 \%$ B3stata).

Tabela 1. Médias anuais, desvio padrão e médias dos meses de novembro de todos os termos de busca.

\begin{tabular}{lrrrrrr}
\hline \multicolumn{1}{c}{ Vol. relativo de buscas } & $\mathbf{2 0 1 4}$ & $\mathbf{2 0 1 5}$ & $\mathbf{2 0 1 6}$ & $\mathbf{2 0 1 7}$ & $\mathbf{2 0 1 8}$ & $\mathbf{2 0 1 9}$ \\
\hline & \multicolumn{7}{c}{ Câncer de próstata } \\
\hline Média anual & 40,7 & 34,7 & 33,8 & 35,5 & 37,2 & 31,6 \\
(desvio padrão) & $(17.2)$ & $(9,6)$ & $(9,6)$ & $(9,7)$ & $(9,2)$ & $(2,4)$ \\
Média Novembro Azul & 59,2 & 64,6 & 63,3 & 66 & 70,3 \\
\hline \multicolumn{7}{c}{ Exame de próstata } \\
\hline Média anual & 31,6 & 25,9 & 25 & 24,4 & 23,6 & 18,4 \\
(desvio padrão) & $(13,4)$ & $(7,3)$ & $(8,3)$ & $(7,1)$ & $(6,5)$ & $(2,8)$ \\
Média Novembro Azul & 57,8 & 55,4 & 55,5 & 55,8 & 55,8 & \\
& \multicolumn{7}{c}{ PSA } \\
\hline Média anual & 44,5 & 45,9 & 47,7 & 54,6 & 63,5 \\
(desvio padrão) & $(8,3)$ & $(6,9)$ & $(7,6)$ & $(9,5)$ & $(9,3)$ & $(6,1)$ \\
Média Novembro Azul & 57 & 63,2 & 77 & 91,3 & 94,8 \\
\hline
\end{tabular}

pronunciado nas buscas sobre "PSA" - os picos de novembro são menos pronunciados em relação aos demais meses. O volume máximo das buscas direcionadas a "câncer de próstata" se dá entre a última semana de outubro e a primeira de novembro de 2015 (63a semana da série); o volume máximo de "exame de próstata" aparece entre a última semana de outubro e a primeira de novembro de 2016 (116a semana) e os maiores VPR voltados ao "PSA" aparecem na terceira semana de novembro de $2017\left(171^{\mathrm{a}}\right)$ e na primeira semana de novembro de 2018 (221 a).

Quanto às médias anuais, "câncer de próstata” se manteve relativamente estável com peque- 


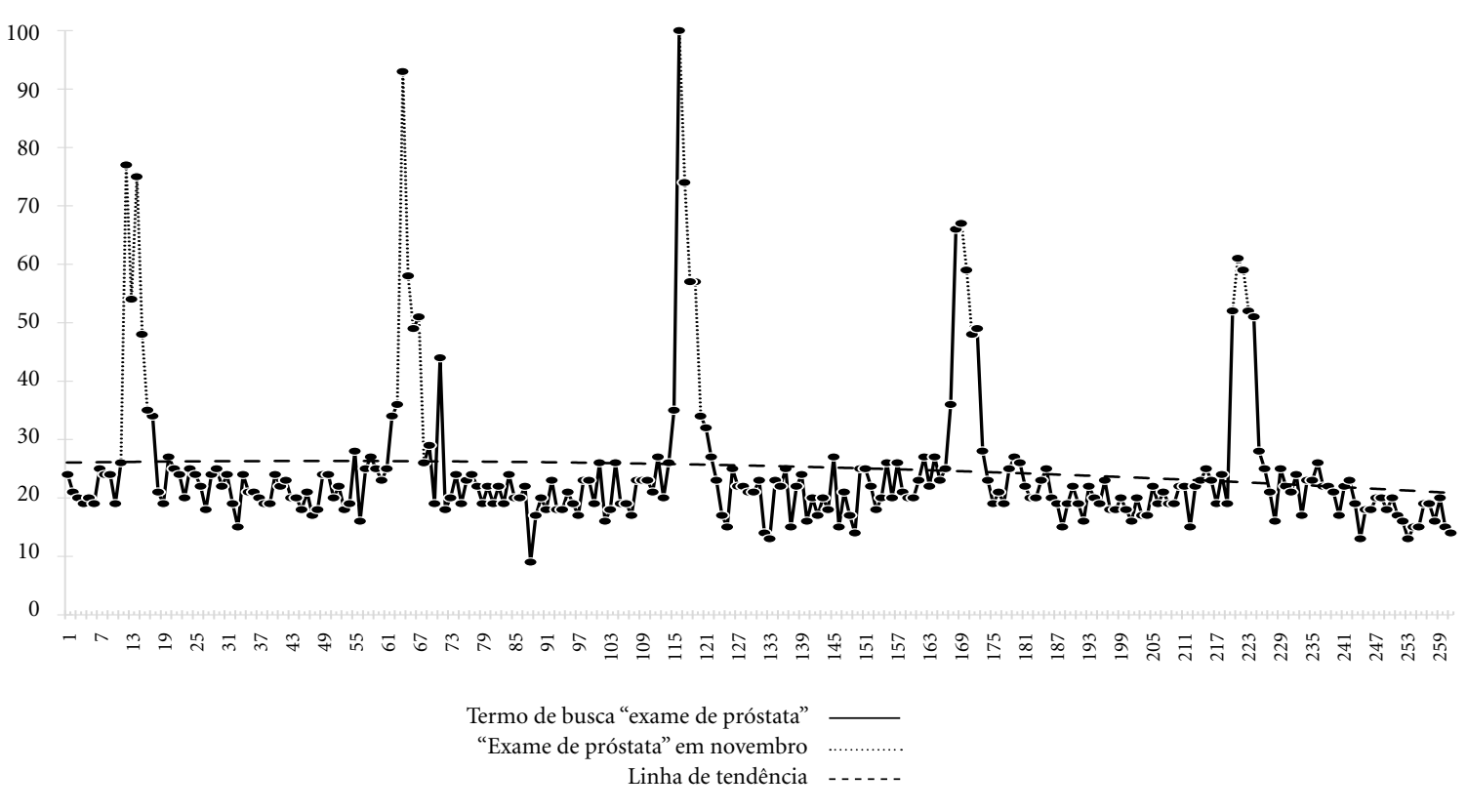

Gráfico 2. Oscilações do termo "exame de próstata”.

Fonte: Google Trends (https://trends.google.com/trends/explore?cat=45\&date=today\%205-y\&geo=BR\&q=exame\%20de\%20pr\%C3\%B3stata).

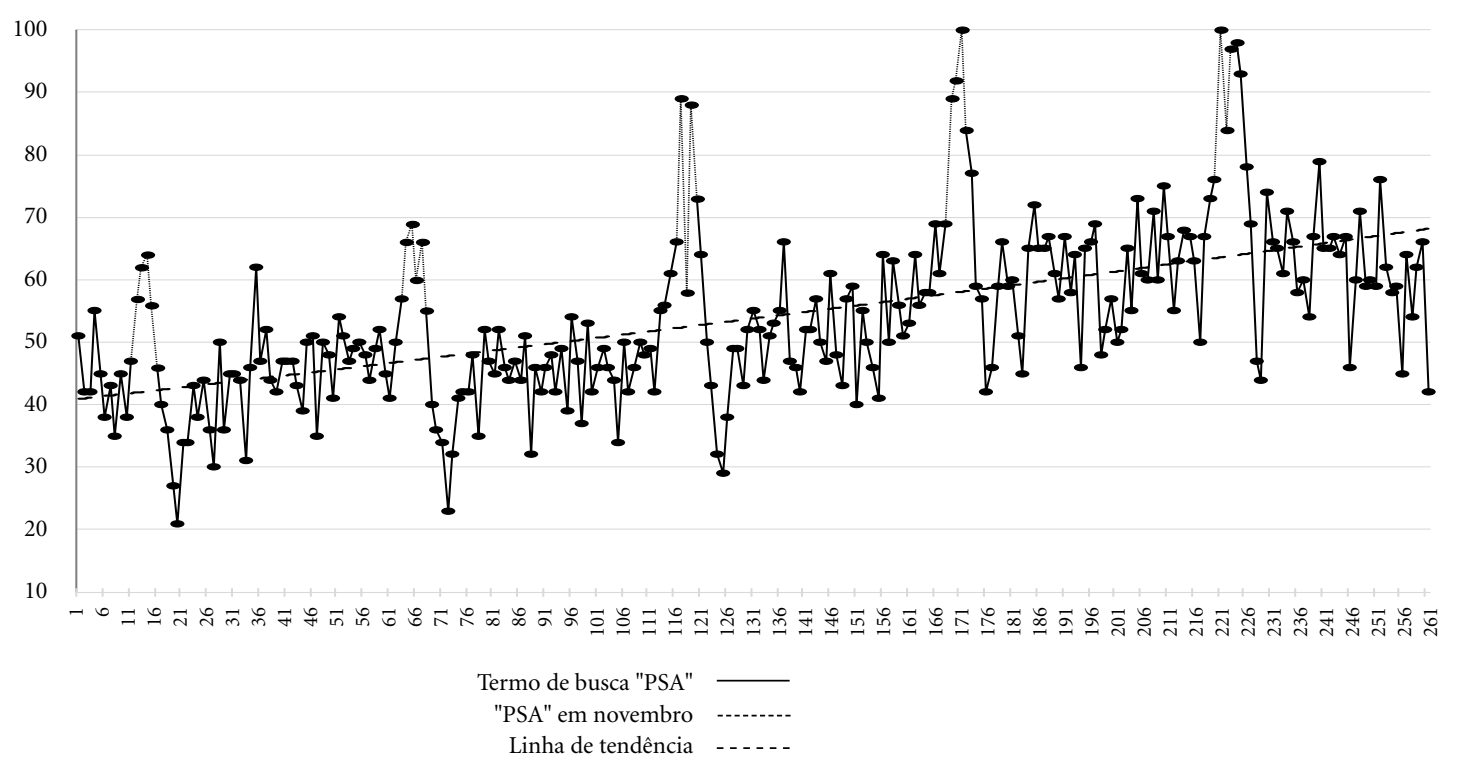

Gráfico 3. Oscilações do termo "PSA".

Fonte: Google Trends (https://trends.google.com/trends/explore?cat=45\&date=today\%205-y\&geo=BR\&q=psa), 
nas variações - o maior percentual de buscas se deu em 2014 com 40,7\% dos acessos; "exame de próstata” entrou em discreta queda (VPR máximo em 2017 - 31,6\%); e "PSA" mostra franca ascensão ao longo dos cinco anos (VPRs máximos em 2019 com média anual de 60,4\%) (Tabela 1). Devido ao recorte do estudo, os anos de 2014 e 2019 devem ser considerados atípicos nos cálculos das médias anuais. A influência dos picos do Novembro Azul nas médias pode ser claramente percebida nas diferenças entre os desvios-padrão de 2014 e 2019 (Tabela 1). Em 2014, sem os sete primeiros meses, $\mathrm{dP}=17,2$. Em 2019, contados apenas os oito primeiros meses, $\mathrm{dP}=2,4$, por excluir o pico do Novembro Azul.

Em relação aos cinco picos de novembro (2014-2018), percebe-se tendência de elevação de "câncer de próstata" (59,2; 64,6; 63,3; 66; e $70,3 \%$, respectivamente), relativa estabilidade em "exame de próstata" (57,8\%; 55,4\%; 55,5\%; $55,8 \%$; e 55,8\%, respectivamente) e marcada elevação de "PSA" (57\%; 63,2\%; 77\%; 91,3\%; e $94,8 \%$, respectivamente) (Tabela 1). Os gráficos 2 e 3 se referem à evolução das buscas relativas voltadas a esses dois últimos termos em separado, sem a interferência de comparação com "câncer de próstata".

\section{Discussão}

No presente estudo, os registros de centenas de milhares de buscas no Google por esclarecimentos sobre assuntos ligados ao câncer de próstata foram analisados como um proxy do interesse coletivo pelo assunto. Pelo que se observa na literatura recente, tal forma de abordagem é amplamente empregada em todo o mundo no que se refere a temáticas assemelhadas ${ }^{17}$. Embora sujeitos a ciclos anuais e regionais, os resultados parecem apontar para um aumento expressivo e persistente do interesse coletivo acerca da prevenção e do diagnóstico do câncer plenamente alinhado à descrição de outros autores ${ }^{13,28-31}$. Tais produtos de pesquisa fornecem abundante material para hipóteses e análises que podem vir a nortear o planejamento de campanhas de promoção de saúde. Nesse cenário, é interessante observar que determinados padrões dos ciclos de interesse se repetem. Um dos mais globalmente recorrentes diz respeito às buscas voltadas a termos ligados a doenças, hábitos e atitudes de prevenção enfatizados por campanhas.

De forma geral, ao lado da descrição do problema (natureza, formas de instalação e desenvolvimento da doença), as mensagens institucionais ressaltam as alternativas acessíveis para evitá-lo ou equacioná-lo (fatores de risco, mudanças nos hábitos e recursos do sistema de saúde para diagnóstico e tratamento). Ocorre que, independentemente da multiplicidade de configurações culturais que povoam o planeta, a curiosidade sobre a prevenção-equacionamento é sempre suplantada por larga margem pela procura por informações sobre a descrição do problema-doença ${ }^{32}$, assim como por alternativas de autodiagnóstico doméstico $^{33}$ ou curas mágicas acessíveis on-line ${ }^{25}$.

Tanto no Brasil como em boa parte do mundo latino, a estratégia de planejamento do Novembro Azul tem sido estruturada com o objetivo de estimular o interesse coletivo pela identificação precoce do câncer de próstata em um contexto de tabus culturais ligados ao exame clínico. De fato, em vista dos dados apresentados, a curiosidade sobre o câncer de próstata parece se instalar episodicamente ao longo dos últimos anos, sem que se observe um interesse estável, homogêneo e determinado na direção do diagnóstico da doença em suas fases iniciais. Assim como no caso em questão, o interesse pelo câncer de mama também cresce em paroxismos anuais impulsionados pelo Outubro Rosa, em escala desproporcionalmente superior se comparada às buscas por dados sobre os procedimentos de rastreio.

Não obstante, pela primeira vez na série histórica disponibilizada pelo GT, em outubro de 2016 o pico de buscas por "mamografia" suplantou o de "câncer de mama", demonstrando expressiva tendência de crescimento ao longo dos meses ${ }^{13}$. Tanto no caso do rastreio do câncer de mama como, de forma geral, em relação ao comportamento de buscas por informações voltadas ao autocuidado, os resultados são consistentes com trabalhos que correlacionam o uso da Internet e a proliferação dos smartphones como impulsionadores de novos comportamentos em uma população com nível de escolaridade crescente, cada vez mais atenta às informações acerca da preservação da saúde. Sabe-se que o nível educacional de uma sociedade (inclusive no Brasil) está diretamente relacionado ao uso cada vez mais frequente do Google a serviço das questões sobre ciência, tecnologia e saúde ${ }^{33-37}$. Além disso, a cobertura midiática e a inaudita capacidade de resgatar informações com rapidez pela Internet também tem favorecido o acesso a informações sobre doenças de celebridades, o que parece alavancar intensamente o interesse pelas doenças e, talvez, por sua prevenção ${ }^{11,38}$.

Com relação a esse último ponto, alguns aspectos culturais ligados a questões de gênero talvez influenciem o efeito-celebridade no que 
concerne às buscas por informações a respeito do rastreamento de câncer de mama, se comparado ao de próstata. Mulheres célebres parecem não se opor à divulgação de seus diagnósticos (usualmente associados a uma espécie de sentença do destino à qual é adequado responder com coragem e serenidade $)^{39,40}$, em contraste com homens famosos que só expõem cânceres de próstata na mídia em casos extremos da evolução da doença. Ainda no terreno das influências culturais de gênero, também é possível levantar outra questão - por que o aumento do nível educacional entre os homens não os estimula a buscar informações na Internet sobre recursos de prevenção e diagnóstico precoce ligados a cânceres masculinos? Isso pode ser facilmente verificado ao Google Trends se compararmos as buscas pelos termos "mamografia", "exame de PSA" e "exame de próstata”. Certamente essa questão transcende aos objetivos do presente trabalho, embora saibamos que a curiosidade direcionada à tecnologia dos diagnósticos é ubíqua entre os gêneros, as faixas etárias do adulto e os níveis socioeconômicos e de escolaridade. Talvez isso esclareça o porquê do interesse acerca do exame sorológico de PSA - seria mera curiosidade sobre uma forma de evitar o exame clínico da próstata que, intimamente, já se admita como necessário? A rejeição cultural dos homens ao exame clínico da próstata acrescentaria, assim, desafios suplementares aos planejadores de campanhas institucionais, embora já se saiba que o nível de escolaridade associado ao acesso à Internet por smartphones favoreçam a adesão e aderência dos homens aos hábitos de rastreamento precoce de doenças ${ }^{34,37}$.

\section{Conclusões, limitações e vantagens do método}

O estudo de tendências de buscas no Google decerto não substitui a acurácia de sistemas de informação sobre screening já presentes em outros países. Há extrema complexidade e custos inacessíveis envolvidos no desenvolvimento e implantação de sistemas nacionais para produção de dados a respeito de aspectos geográfico-culturais ligados à prevenção de doenças em um país de dimensões continentais. Além disso, há escassez de métodos precisos para avaliação das campanhas institucionais que apontem tendências vigentes e indícios de falhas ou sucessos ao longo dos anos. Por outro lado, os resultados aqui expostos descrevem de forma eloquente o crescente interesse potencializado pelo estímulo das mídias e o acesso facilitado a informações sobre cuidados à saúde.

Certamente os resultados apresentados não esclarecem de forma inequívoca se as campanhas para rastreio do câncer seguem por uma trilha exitosa. Não obstante, torna-se evidente que há recursos plenamente disponíveis e pouco onerosos disponibilizados pelas tecnologias de informação que acenam com a possibilidade de novas metodologias de identificação de tendências. É importante perceber que o conceito de "tendência" (em complementação ao de "incidência" e "prevalência") talvez acrescente novas perspectivas ao cenário da saúde coletiva - até aqui instrumentalizada unicamente pela constatação a posteriori dos fenômenos quantificáveis mais relevantes.

\section{Colaboradores}

PR Vasconcellos-Silva trabalhou na concepção, delineamento, análise, interpretação dos dados, redação do texto e em sua revisão crítica. TC Araújo-Jorge trabalhou na redação do texto e em sua revisão crítica. 


\section{Referências}

1. Câncer de próstata. Instituto Nacional de Câncer. Ministério da Saúde [página na Internet]. [acessado 2019 Set 03]. Disponível em: https://www.inca.gov.br/ tipos-de-cancer/cancer-de-prostata.

2. Djulbegovic M, Beyth RJ, Neuberger MM, Stoffs TL, Vieweg J, Djulbegovic B, Dahm P. Screening for prostate cancer: systematic review and metaanalysis of randomised controlled trials. BMJ 2010; 341:c4543.

3. Wever EM, Draisma G, Heijnsdijk EAM, Roobol MJ, Boer R, Otto SJ, de Koning HJ. Prostate-Specific Antigen Screening in United States vs in the European Randomized Study of screening for prostate cancer Rotterdam. JNCI 2010; 102(5):352-355.

4. Carroll PR, Vickers AJ. Point/counterpoint: early detection of prostate cancer: do the benefits outweigh the consequences? J Natl Compr Canc Netw 2014; 12(Supll. 5):768-771.

5. Valera P, Lian Z, Brotzman L, Reid A. Fatalistic cancer beliefs and information seeking in formerly incarcerated African-American and Hispanic men: implications for cancer health communication and research. Health Commun 2018; 33(5):576-84.

6. Balancing the benefits and risks of choice. Lancet 2016; 388(10050):1129.

7. Bansal A, Koepl LM, Fedorenko CR, Li C, Smith JL, Hall IJ, Penson DF, Ramsey SD. Information seeking and satisfaction with information sources among spouses of men with newly diagnosed local-stage prostate cancer. J Cancer Educ 2018;33(2):325-331.

8. Nam S, Han SH, Gilligan M. Internet use and preventive health behaviors among couples in later life: evidence from the health and retirement study. Gerontologist 2019; 59(1):69-77.

9. Bravo CA, Hoffman-Goetz L. Tweeting About Prostate and Testicular Cancers: What Are Individuals Saying in Their Discussions About the 2013 November Canada campaign. J Cancer Educ 2016; 31(3):559-566.

10. Bravo CA, Hoffman-Goetz L. Social media and men's health: a content analysis of twitter conversations during the 2013 November campaigns in the United States, Canada, and the United Kingdom. Am J Mens Health 2017; 11(6):1627-1641.

11. Vasconcellos-Silva PR, Sormunen T, Craftman ÅG. Evolution of accesses to information on breast cancer and screening on the Brazilian National Cancer Institute website: an exploratory study. Cien Saude Colet 2018; 23(4):1303-1312.

12. Saab MM, Reidy M, Hegarty J, O’Mahony M, Murphy M, Von Wagner C, Drummond FJ. Men's information-seeking behavior regarding cancer risk and screening: a meta-narrative systematic review. Psychooncology 2018; 27(2):410-419.

13. Vasconcellos-Silva PR, Carvalho DBF, Trajano V, de La Rocque LR, Sawada ACMB, Juvanhol LL. Using Google Trends data to study public interest in breast cancer screening in Brazil: why not a Pink February? JMIR Public Health Surveill 2017; 3(2):e17.

14. Black PC, Penson DF. Prostate cancer on the internet-information or misinformation? J Urol 2006; 175:1836-1842.
15. Manole BA, Wakefield DV, Dove AP, Dulaney CR, Marcrom SR, Schwartz DL, Farmer MR. Quality of prostate cancer screening information on the websites of nationally recognized cancer centers and health organizations. Pract Radiat Oncol 2018; 8(4):275-278.

16. Schootman M, Toor A, Cavazos-Rehg P, Jeffe DB, McQueen A, Eberth J, Davidson NO. The utility of Google Trends data to examine interest in cancer screening. BMJ Open 2015; 5(6):e006678.

17. Nuti SV, Wayda B, Ranasinghe I, Wang S, Dreyer RP, Chen SI, Murugiah K. The use of Google Trends in health care research: a systematic review. PLoS ONE 2014; 9(10):e109583.

18. Ginsberg J, Mohebbi MH, Patel RS, Brammer L, Smolinski MS, Brilliant L. Detecting influenza epidemics using search engine query data. Nature 2009; 457(7232):1012-1014.

19. Guo P, Zhang J, Wang L, Yang S, Luo G, Deng C, Wen $\mathrm{Y}$, Zhang Q. Monitoring seasonal influenza epidemics by using internet search data with an ensemble penalized regression model. Sci Rep 2017; 7:46469.

20. Phillips CA, Barz-Leahy A, Li Y, Schapira MM, Bailey LC, Merchant RM. Relationship between state-level Google online search volume and cancer incidence in the United States: retrospective study. J Med Internet Res 2018; 20(1):e6.

21. Passos JA, Vasconcellos-Silva PR, Santos LAS. Ciclos de atenção de dietas da moda e tendências de busca na internet pelo Google Trends. Cien Saude Colet 2020; 25(7):2615-2631.

22. Tijerina JD, Morrison SD, Nolan IT, Vail DG, Nazerali R, Lee GK. Google Trends as a tool for evaluating public interest in facial cosmetic procedures. Aesthet Surg J 2019; 39(8):908-918.

23. Silva PR, Castiel LD, Ferreira FR. Patterns of cancer -related internet searches: reactiveness; risks; the role of affect. Cien Saude Colet 2016; 21(3):861-870.

24. Choi H, Varian H. Predicting the present with Google Trends. Technical report, Google, 2009 [página na Internet]. [acessado 2019 Ago 15]. Disponível em: http://google.com/googleblogs/pdfs/google_predicting_the_present.pdf.

25. Vasconcellos-Silva PR, Castiel LD, Bagrichevsky M, Griep RH. New information technologies and health consumerism. Cad Saude Publica 2010; 26(8):14731482.

26. Conselho Nacional de Saúde. Resolução no 510 , de 7 de abril de 2016 [documento na Internet]. [acessado 2019 Ago 20]. Disponível em: http://conselho.saude. gov.br/resolucoes/2016/Reso510.pdf.

27. WMA Declaration of Helsinki - Ethical Principles for Medical Research Involving Human Subjects, 2018 [documento na Internet]. [acessado 2019 Set 05]. Disponível em: https://www.wma.net/policies-post/ wma-declaration-of-helsinki-ethical-principles-formedical-research-involving-human-subjects.

28. Cacciamani GE, Bassi S, Sebben M, Marcer A, Russo GI, Cocci A et al. Consulting "Dr. Google" for prostate cancer treatment options: a contemporary worldwide trend analysis. Eur Urol Oncol 2019; pii: S25889311(19)30105-1. 
29. Rezaee ME, Goddard B, Sverrisson EF, Seigne JD, Dagrosa LM. 'Dr Google': trends in online interest in prostate cancer screening, diagnosis and treatment. BJU Int 2019; DOI: 10.1111/bju.14846. [epub ahead of print].

30. Bloom R, Amber KT, Hu S, Kirsner R. Google Search Trends and skin cancer: evaluating the US population's interest in skin cancer and its association with melanoma outcomes. JAMA Dermatol 2015; 151(8):903-905.

31. Kantor J. Association of Google search volume index peaks for skin cancer with skin cancer awareness month. JAMA Dermatol 2016; 152(1):113.

32. Vasconcellos-Silva PR, Castiel LD, Griep RH, Zanchetta M. Cancer prevention campaigns and Internet access: promoting health or disease? J Epidemiol Community Health 2008; 62(10):876-881.

33. Vasconcellos-Silva PR, Castiel LD. New self-care technologies and the risk of self-diagnosis through the Internet. Rev Panam Salud Publica 2009; 26(2):172-175.

34. Jiang S, Street RL. Pathway linking internet health information seeking to better health: a moderated mediation study. Health Commun 2017; 32(8):10241031.

35. Cao W, Zhang X, Xu K, Wang Y. Modeling online health information-seeking behavior in China: the roles of source characteristics, reward assessment, and internet self-efficacy. Health Commun 2016; 31(9):11051114.

36. Portal Brasil. Cidadania e Justiça. Women's education increases relative to men [página na Internet]. [acessado 2019 Ago 27]. Disponível em: http://www.brasil. gov.br/cidadania-e-justica/2014/11/escolaridade-das -mulheres-aumenta-em-relacao-a-dos-homens.

37. Segev E, Baram-Tsabari A. Seeking science information online: data mining Google to better understand the roles of the media and the education system. $P u$ blic Underst Sci 2012; 21(7):813-829.

38. Cooper CP, Gelb CA, Lobb K. Celebrity appeal: reaching women to promote colorectal cancer screening. J Womens Health (Larchmt) 2015; 24(3):169-173.

39. Vos SC, Sutton J, Gibson CB, Butts CT. Celebrity cancer on Twitter: mapping a novel opportunity for cancer prevention. Cancer Control 2019; 26(1):1073274819825826.

40. Evans DG, Barwell J, Eccles DM, Collins A, Izatt L, Jacobs C, Donaldson A, Brady AF, Cuthbert A, Harrison R, Thomas S, Howell A, The FH02 Study Group, RGC teams, Miedzybrodzka Z, Murray A. The Angelina Jolie effect: how high celebrity profile can have a major impact on provision of cancer related services. Breast Cancer Res 2014; 16(5):442.

Artigo apresentado em 05/09/2019

Aprovado em 28/10/2019

Versão final apresentada em 30/10/2019

Editores-chefes: Romeu Gomes, Antônio Augusto Moura da Silva 
